\title{
Advances in Hypoxia-Mediated Mechanisms in Hepatocellular Carcinoma
}

\author{
Xin Xin Xiong, Xin Yao Qiu, Dian Xing Hu, and Xiao Qian Chen \\ Department of Pathophysiology, School of Basic Medicine, Huazhong University of Science and Technology, Wuhan, China
}

Received December 1, 2016; accepted February 21, 2017

\begin{abstract}
Hepatocellular carcinoma (HCC) is the fifth most common and the third most deadly malignant tumor worldwide. Hypoxia and related oxidative stress are heavily involved in the process of HCC development and its therapies. However, direct and accurate measurement of oxygen concentration and evaluation of hypoxic effects in HCC prove difficult. Moreover, the hypoxia-mediated mechanisms in HCC remain elusive. Here, we summarize recent major evidence of hypoxia in HCC lesions shown by measuring partial pressure of oxygen $\left(\mathrm{pO}_{2}\right)$, the clinical importance of hypoxic markers in HCC,
\end{abstract}

and recent advances in hypoxia-related mechanisms and therapies in HCC. For the mechanisms, we focus mainly on the roles of oxygen-sensing proteins (i.e., hypoxia-inducible factor and neuroglobin) and hypoxia-induced signaling proteins (e.g., matrix metalloproteinases, high mobility group box 1, Beclin 1, glucose metabolism enzymes, and vascular endothelial growth factor). With respect to therapies, we discuss mainly YQ23, sorafenib, 2-methoxyestradiol, and celastrol. This review focuses primarily on the results of clinical and animal studies.

\section{Introduction}

Liver cancer is presently the fifth most prevalent malignant tumor and the third leading cause of cancer-related death worldwide. Primary hepatocellular carcinoma (HCC) or malignant hepatoma accounts around $80 \%$ of liver cancers. In the U.S., it was estimated that there were approximately 33,190 newly diagnosed liver cancer cases and 23,000 liver cancer deaths in the year 2014, representing a steady rise in liver cancer incidence (1975-2010) and mortality (1992-2010) (Siegel et al., 2014). In China, the incidence and mortality of liver cancers have increased rapidly and the number of liver cancer deaths was ranked in the top two among all types of cancers in 2014 (McGuire, 2016). The high mortality of liver cancers reflects the general ineffectiveness of current HCC therapies.

Epidemiologically, HCC occurs mainly in Asia, sub-Saharan Africa, North America, and Western Europe and is correlated with the prevalence of hepatitis B virus (in China) or hepatitis

This work was supported by funds from the National Nature Science Foundation of China (Grants 81172397, 81471386, and 81672504).

https://doi.org/10.1124/mol.116.107706.
C virus (in Japan), or with infection and alcoholism (in Western Europe). Aflatoxin and liver cirrhosis are also important risk factors for HCC (Zhang et al., 2015). The development of HCC is a complicated pathologic process in which hypoxia and related oxidative stress are common pathophysiological factors associated with infection/inflammation or cellular toxicity/injury after exposure to various risk factors (Severi et al., 2010). Chronic hypoxia and related oxidative stress induce profound epigenetic/genetic alterations in hepatocytes, accompanying repetitive-injury regeneration of hepatocytes upon risk factor exposure. These responses lead to cellular stress adaptation and ultimately to HCC carcinogenesis (Nishida and Kudo, 2013).

HCC is treated mainly by surgical resection, liver transplantation, chemotherapy (e.g., sorafenib), interventional chemotherapy [e.g., transcatheter arterial chemoembolization (TACE)], and/or radiation. Hypoxia or ischemia always accompany and then follow HCC therapies and heavily affect the therapeutic outcomes. In HCC interventional therapies, ischemic death is a major therapeutic mechanism. In addition, hypoxic responses to therapy have important prognostic value for HCC (Hao et al., 2013; Hayano et al., 2014; Ippolito et al., 2014).

ABBREVIATIONS: 2-ME2, 2-Methoxyestradiol; CA-IX, carbonic anhydrase IX; CT, computerized tomography; Cygb, cytoglobin, DFS, disease-free survival; EGFR, epidermal growth factor receptor; GLUT1, glucose transporter 1; H2AX, H2A histone family, member X; $\gamma$-H2AX, phosphorylated H2AX; HCC, hepatocellular carcinoma; HIF- $1 \alpha / 2 \alpha$, hypoxia inducible factor- $1 \alpha / 2 \alpha$; HMGB1, high-mobility group box 1 protein; IL, interleukin; miR, microRNA; LSD1, lysine-specific demethylase-1; MMPs, matrix metalloproteinases; mtDNA, mitochondrial DNA; NF, nuclear factor; Ngb, neuroglobin; OS, overall survival; PFS, progression-free survival; PK, pyruvate kinase; PTEN, phosphatase and tensin homolog; SDF1, stromal cellderived factor-1; STAT, signal transducer and activator of transcription; TACE, transcatheter arterial chemoembolization; TIMP, tissue inhibitor of metalloproteinases; TLR, Toll-like receptor; TNM, tumor-node-metastasis; VEGF, vascular endothelial growth factor. 
Hypoxia is considered to be involved in HCC development and therapy; however, the exact roles hypoxia plays remain elusive. The major reasons include the following: 1) direct measurement of partial pressure of oxygen $\left(\mathrm{pO}_{2}\right)$ in patient HCC lesions is rare; 2 ) there is no specific signaling molecule or receptor for hypoxia; and 3) hypoxic responses are extremely complicated. In this review, we first discuss the direct evidence and clinical significance of hypoxia in HCC lesions. We then summarize most recent advances in hypoxia-related mechanisms and therapies in HCC.

\section{Direct Evidence of Hypoxia in HCC Lesions by Measuring $\mathrm{pO}_{2}$}

Pathophysiologically, hypoxia refers to insufficient oxygen supply to a cell/tissue/organ or impaired cellular oxygen utilization that finally results in malfunction of the cell/ tissue/organ. The liver accepts both oxygenated blood from the hepatic artery $(\sim 30 \%)$ and deoxygenated blood from the portal vein $(\sim 70 \%)$. The oxygen tensions of the influent and effluent blood in the hepatic sinusoid (supplying oxygen to hepatocytes) are 60-65 and 30-35 $\mathrm{mmHg}$, respectively, lower than that of most other tissue capillaries (74-104 and 34-46 mmHg) (Jungermann and Kietzmann, 2000). Further, blood flow in the hepatic sinusoid is relatively slow, whereas the metabolic rate of hepatocytes is relatively high. Therefore, hepatocytes are particularly vulnerable to primary or secondary hypoxia after viral infection, toxic substances exposure, or inflammation. Li et al. (2015) reported that the liver is one of the three organs most vulnerable to hypoxia, as determined by exposing rats to high-altitude anoxia $\left(\mathrm{pO}_{2} 20 \mathrm{mmHg}\right)$ compared with sea level $\mathrm{pO}_{2}$ (83 $\left.\mathrm{mmHg}\right)$. Upon exposure to endotoxin, mouse liver sinusoidal $\mathrm{pO}_{2}$ was reduced by $75 \%$ from 5 to $44 \mathrm{mmHg}$ (15 minutes) to $11 \mathrm{mmHg}$ ( 6 hours), although cardiac output, arterial oxygen saturation, and blood flow in the hepatic artery were unaffected (James et al., 2002). In patients with liver cirrhosis, hypoxemia ranges from 10 to $40 \%$ depending on hepatic dysfunction (Møller et al., 1998). Hypoxia profoundly interferes with immune responses and cell survival/death machinery and induces epigenetic/proteomic/genomic alterations as a result of the reduced $\mathrm{pO}_{2}$ level associated with cell malignancy (Höckel and Vaupel, 2001). After exposing mouse tumor cells to $\mathrm{pO}_{2}$ of $<1 \mathrm{mmHg}$ for 4 hours, the mutation rate was increased by 3.4-fold compared with the normoxic control (Reynolds et al., 1996). Since the liver is frequently exposed to various toxic insults, and hypoxia can be easily induced in the liver after injury, it is conceivable that repetitive or chronic hypoxia occurs during pathologic situations such as liver cirrhosis. Considering the prominent effect of hypoxia in inducing gene mutation, repetitive or chronic hypoxia might be a driving force for carcinogenesis of hepatocytes.

During the process of tumor development, cancer cells inside a solid tumor can suffer from three major types of hypoxia, chronic diffusion hypoxia, acute/intermittent perfusion hypoxia, or/and anemic hypoxia, owing to the abnormal growth of tumor vasculature, increased tumor size, and reduced oxygen concentration in the blood. A precise diagnosis of intratumor hypoxia is difficult in patients, particularly at the cellular level. Hypoxia is associated with many factors, including peripheral $\mathrm{pO}_{2}$, oxygen saturation/content in capillaries, tissue blood perfusion volume, the distance between cells and capillaries, amount and quality of cellular mitochondria, and oxygen demand of cells. Among all hypoxic parameters, intratumor $\mathrm{pO}_{2}$ is the most direct indicator of tumor hypoxia (Höckel and Vaupel, 2001).

At the initial stage of development of a solid tumor, cancer cell proliferation overwhelms tumor angiogenesis. Therefore, cancer cells approximately $70 \mu \mathrm{m}$ away from the oxygenated blood may suffer from diffusion hypoxia since oxygen diffusion decreases within 100-200 $\mu \mathrm{m}$ of a functional capillary. Liu et al. (2014) examined tumor oxygenation by using OxyLab $\mathrm{pO}_{2}$ in an orthotropic rat $\mathrm{HCC}$ model and found that most regions inside the tumor $(74.1 \%)$ had $\mathrm{pO}_{2}$ values of 0-10 mmHg. When $\mathrm{pO}_{2}$ was measured using a fluorescence fiberoptic oxygen probe, it was found that the $\mathrm{pO}_{2}$ inside tumors ranged from 0.2 to $0.8 \mathrm{mmHg}$ in three rat liver tumors with diameters of $0.7,1.0$, and $1.5 \mathrm{~cm}$; this $\mathrm{pO}_{2}$ value was significantly lower than that in normal liver tissue (45 mmHg) (Riedl et al., 2008). These finding are consistent with the fact that intratumor $\mathrm{pO}_{2}$ is severely decreased in other types of solid cancers (e.g., primary uterine cervix cancers) at their early stages (Vaupel et al., 2007). Direct intratumor polarographic measurement of $\mathrm{pO}_{2}$ is the "gold standard" method for measuring intratumor hypoxia. In four patients with liver metastases of rectal cancers, the measured median $\mathrm{pO}_{2}$ inside tumors was $6 \mathrm{mmHg}$, much lower than that in normal liver tissues $(30 \mathrm{mmHg}$ ) (Vaupel et al., 2007). Polarography data measuring $\mathrm{pO}_{2}$ in primary $\mathrm{HCC}$ are rare.

During development of a tumor, tumor angiogenesis is prominent and is induced mainly by hypoxia-induced vascular endothelial growth factor (VEGF) produced in cancer cells. The vasculature in HCC is reconstructed profoundly from dominant portal perfusion to dominant hepatic arterial perfusion, causing larger HCC to become perfusion-rich tumors (Chou et al., 2014). Taouli et al. (2013) reported an increase in the arterial blood flow/fraction and a decrease in portal venous blood flow in HCC (tumor size: 1.1-12.6 cm, $n=$ 26), compared with liver parenchyma, by dynamic contrastenhanced magnetic resonance imaging (DCE-MRI). Similarly, Chen et al. (2016) reported that advanced HCC had significantly higher peak (maximal perfusion signal intensity), slope (maximal ascending slope of perfusion curve), arterial fraction (hepatic artery/portal vein perfusion), and arterial flow, but lower portal flow, distribution volume, and mean transit time compared with surrounding liver parenchyma $(n=92)$ as measured by DCE-MRI. The overall survival (OS) of patients with higher peak blood flow in their tumors is significantly improved, suggesting that hypoxia is an adverse factor for HCC patient survival. Similar results were also reported from studies using perfusion computerized tomography (CT) detection (Arizumi et al., 2014; Bayraktutan et al., 2014; Guo and $\mathrm{Yu}, 2014$ ). Owing to the abnormal vessel structure or function, acute/intermittent perfusion hypoxia is common in HCC. It is generally believed that heterogeneous microregional hypoxia is widely distributed inside HCC, as has been shown in other types of cancers (Vaupel et al., 2007). Guo and $\mathrm{Yu}$ (2014) examined the perfusion of tumor tissue in 45 patients with HCC by four-dimensional (4D) CT and found that a cancer-feeding artery was present in only 28 cases. Among these 28 cases, 20 had thickened, rigid, or distorted feeding arteries. Moreover, 14 cases had a thrombus in the portal veins (Guo and Yu, 2014). In another study, regional tumor 
blood flow (20.6-105.7 ml/100 g per minute) and oxygen extraction fraction (20.4-56.7\%)/oxygen metabolic rate (1.71$5.05 \mathrm{ml} / 100 \mathrm{~g}$ per minute) varied greatly between individual $\operatorname{HCC}(n=6)$ as measured by perfusion CT (Fukuda et al., 2004). Together, these studies suggest malfunction of arterial perfusion inside HCC despite the increased arterial perfusion volume. Secondary hypoxia in HCC after treatments such as TACE is common and is an important indicator of therapeutic effectiveness and an independent predictor for HCC prognosis (Hao et al., 2013; Hayano et al., 2014; Ippolito et al., 2014); this topic is beyond the focus of this review. Although measurements of perfusion parameters can indicate tumor hypoxia, measuring $\mathrm{pO}_{2}$ in primary $\mathrm{HCC}$ using an intratumor polarographic oxygen-sensor is indispensable for diagnosing tumor hypoxia in $\mathrm{HCC}$ and will be an important parameter for precise targeting of hypoxia in HCC.

\section{Clinical Importance of Hypoxic Markers in HCC}

Clinically, hypoxia is considered as an independent adverse prognostic factor for HCC. Lai et al. (2015) reported that carbonic anhydrase IX (CA-IX, a hypoxia marker) was expressed in 19 of $40(47.5 \%)$ residual/recurrent HCCs (after TACE) and 2 of 17 (11.8\%) untreated HCCs. Huang et al. (2015) reported that CA-IX was detected in 110 of the $227(48.5 \%)$ unifocal and respectable primary HCC tumors, correlating with younger age, female sex, larger tumor size, higher tumor grade and higher tumor stage. CA-IX-positive HCC patients had a lower 5-year overall survival/disease-free survival (OS/DFS). Furthermore, CA-IX was a poor predictor of DFS and OS in advanced HCC. After analyzing the relationship between hypoxia markers (HIF- $1 \alpha$ and CA-IX) and patient survival parameters (i.e., OS and recurrence-free survival) in 179 primary HCC, Srivastava et al. (2015) demonstrated that high expression levels of HIF- $1 \alpha$ and CA-IX appeared in $60 \%$ and $15 \%$ of cases, respectively, correlating with a worse prognosis independent of antigen Ki-67 expression. When hypoxia markers and Wnt pathway markers were coexpressed, the OS in HCC (tumor size $<5 \mathrm{~cm}$ ) was worse. Similar results regarding the role of HIF- $1 \alpha$ in HCC prognosis were reported by Osman et al. (2015). In this study, HIF- $1 \alpha$ was overexpressed in 42 of 65 HCC specimens (64.6\%), correlating to larger tumor sizes, more tumor loci, and more advanced stages of the disease. Further, the authors found that the expression of autophagy marker Beclin-1 was associated with that of HIF- $1 \alpha$ in HCC. In the high HIF- $1 \alpha$ group, but not low HIF- $1 \alpha$ group, more HCC cells were Beclin1-positive, suggesting that hypoxia might activate autophagy during HCC development. The clinical significance of Beclin-1 expression in HCC will be discussed in the following section. In addition to HCC specimens, HIF- $1 \alpha$ levels were also increased in the serum of patients with HCC compared with healthy populations and with patients with liver cirrhosis, and showed a significant correlation with nuclear factor $(\mathrm{NF})-\kappa \mathrm{B}$ p65 expression/activity and also poor prognosis of HCC (Gaballah et al., 2014). Wang et al. (2014) reported that HIF- $1 \alpha$ mRNA levels $(n=32)$ and protein levels $(n=33)$ were upregulated in HCC lesions compared with adjacent nontumor tissues, correlating with larger tumor sizes, metastasis, advanced disease stage, and shorter survival time. In HCC after liver transplantation, higher HIF- $1 \alpha$ levels were also significantly associated with tumor invasion, advanced TNM stages, as well as shorter OS in 31 patients (Xiao et al., 2014). The authors compared the effect of preoperative TACE with that of non-TACE on HCC prognosis $(n=$ $25)$ and found that in TACE-treated HCC $(n=10)$ an increase in HIF- $1 \alpha$ expression was correlated to an increase in the 2-year recurrence rate and shorter diseasefree survival (Xu et al., 2014). However, a meta-analysis of $851 \mathrm{HCC}$ cases from eight studies by Cao et al. (2014) showed that HIF- $1 \alpha$ protein levels were correlated only with vascular invasion or worse DFS but not to other HCC pathologic characteristics (e.g., capsule formation, cirrhosis, tumor size, and tumor differentiation). Together, the results of these studies support HIF- $1 \alpha$ as a reliable indicator of prognosis for all HCC, whereas CA-IX is still not reliable because its expression in HCC cases varies greatly $(11.8-48.5 \%)$ between different studies.

\section{Hypoxia-Related Mechanisms in HCC}

It is generally believed that hypoxia plays important roles in hepatocarcinogenesis, HCC development, and HCC reoccurrence after chemotherapy by promoting HCC cell proliferation/invasion and angiogenesis. HIF- $1 \alpha$ is the most commonly investigated player in HCC hypoxic responses. HIF-2 $\alpha$, matrix metalloproteinases (MMPs), high mobility group box 1 (HMGB1), Beclin 1, and glucose metabolism enzymes are recently investigated molecules that are also involved in hypoxia-induced effects in HCC. In addition, neuroglobin (Ngb) is a novel intracellular $\mathrm{O}_{2}$-binding protein that may directly sense $\mathrm{pO}_{2}$ changes in HCC cells. These molecules might collaborate to sense and transduce hypoxic signaling in HCC cells during HCC development and therapies (Fig. 1, Table 1).

\section{Role of HIF-1 $\alpha$ in HCC}

HIFs (including HIFs 1, 2, and 3) are heterodimeric transcription factors ( $\alpha$ and $\beta$ subunits) that are responsive to reduced cellular oxygen supply. In the presence of oxygen, HIF prolyl-hydroxylase catalyzes the hydroxylation of HIF- $1 \alpha$ at proline residues, leading to rapid proteasomal degradation of HIF- $1 \alpha$ via a von Hippel-Lindau protein-dependent mechanism. During hypoxia, the activity of HIF prolyl-hydroxylase is inhibited. This allows HIF- $1 \alpha$ to accumulate and bind to HIF- $1 \beta$, forming a stabilized HIF- $1 \alpha / 1 \beta$ dimer. The dimer enters into the nuclei to exert its transcriptional activity by binding to the HIF-responsive elements in promoters (containing NCGTG sequence) of a variety of genes, including glycolysis enzymes and VEGF.

HIF-1 and NF- $\kappa \mathrm{B}$ are inter-regulated transcriptional factors during hypoxia and inflammation. Most studies demonstrate that HIF-1 enhances $\mathrm{NF}-\kappa \mathrm{B}$ transcription mainly during acute hypoxia, whereas NF- $\kappa \mathrm{B}$ promotes the expression of HIF- $1 \alpha$ under normal conditions and in response to inflammatory stimuli [e.g., tumor necrosis factor $\alpha$ and oxygen reactive species (ROS)]. Recently, Jiang et al. (2015) demonstrated that only the p50/p65 subunits of NF- $\kappa$ B upregulated HIF- $1 \alpha$ upon acute hypoxia, whereas the c-Rel subunit of $\mathrm{NF}-\kappa \mathrm{B}$ downregulated HIF- $1 \alpha$ during prolonged hypoxia via miR-93 and miR-199a-5p in HCC cells. Meanwhile, HIF-1 $\alpha$ upregulated Dicer1 [a key enzyme in mature micro-RNA (miR) 


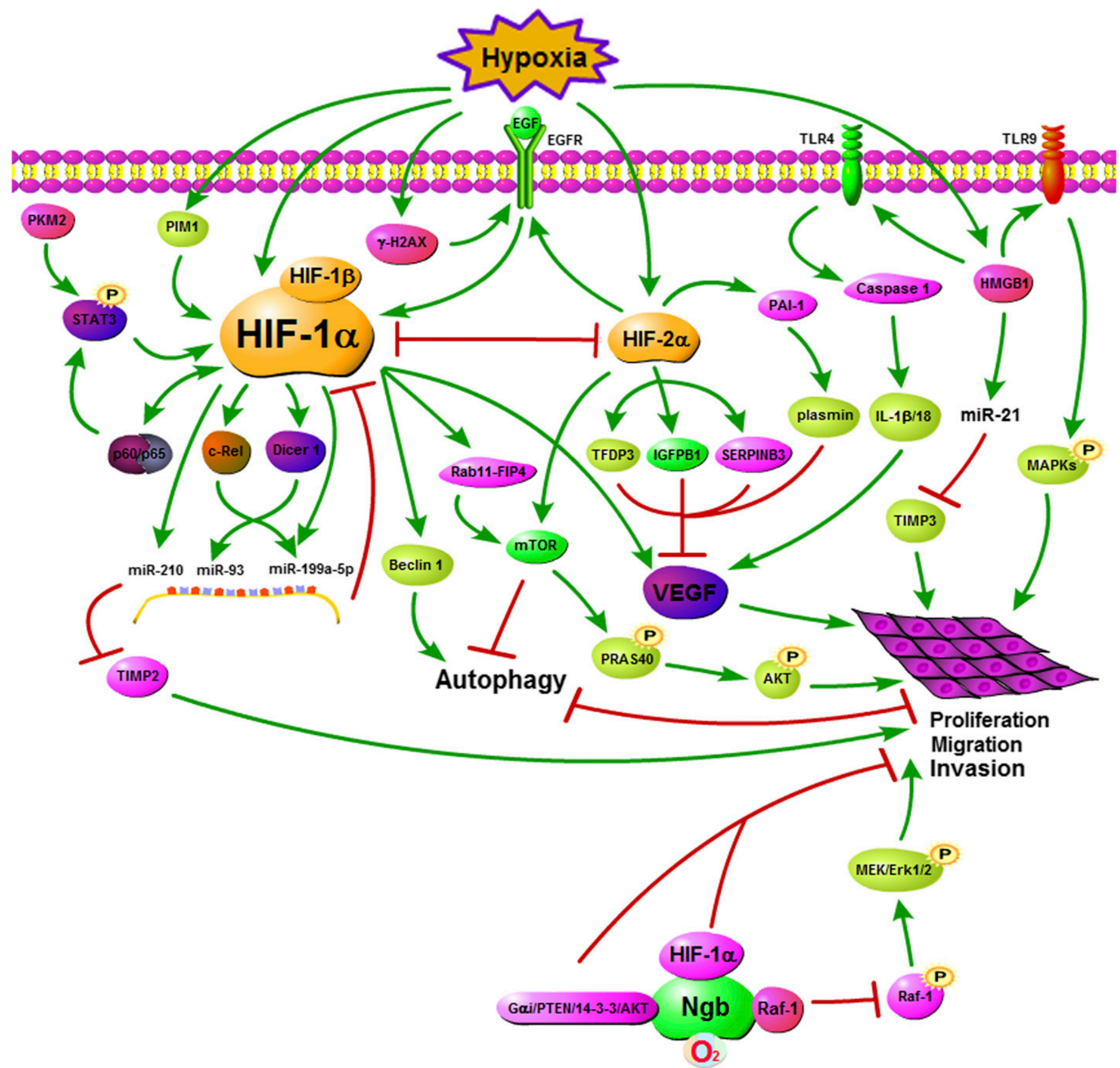

Fig. 1. Hypoxia-related mechanisms in HCC. Hypoxia upregulates/activates HIF-1 $\alpha$, HIF-2 $\alpha, \gamma$-H2AX, PIM1, HMGB1, Rab11-FIP4, PAI-1, SERPNIB3, IGFBP1, MMPs, and TIMP2 either directly or indirectly, which further regulates downstream signaling proteins to promote or suppress HCC cell proliferation, migration, and invasion. In addition, PKM2, EGFR, miRs, TLR4/9, and autophagy play roles in the regulation of hypoxia-mediated signaling pathways. Independently, neuroglobin (Ngb) senses hypoxia signal via binding to $\mathrm{O}_{2}$ and by regulating Raf- 1 , HIF- $1 \alpha$, G $\alpha$ I, AKT, PTEN, and 14-3-3 proteins simultaneously. The binding of Ngb to Raf-1 leads to inactivation of Raf-1-MEK-Erk1/2 pathway and prevents HCC cell proliferation/migration. $\rightarrow$, upregulation/activation; $\perp$, Downregulation/inhibition; 14-3-3, 14-3-3 proteins; AKT, AKT serine/threonine kinase 1; c-Rel, reticuloendotheliosis oncogene C; Dicer 1, dicer 1, ribonuclease III; EGF, epidermal growth factor; Erk1/2, extracellular signal-regulated kinase 1/2; Gai, G-protein alpha subunit; IGFB1, insulin-like growth factor binding protein; MAPK, mitogen-activated protein kinase; MEK, mitogen-activated protein kinase kinase; mTor, mammalian target of rapamycin; P60/p65, protein of 60/65 kDa; PAI-1, plasminogen activator inhibitor 1; PIM1, serine/threonineprotein kinase pim-1; PKM2, pyruvate kinase 2, muscle; PRA S40, proline-rich AKT substrate of $40 \mathrm{kDa}$ or AKT1 substrate 1; PTEN, phosphatase and tensin homolog; Rab11-FIP4, Rab11-family interacting protein 4; SERPINB3, serpin peptidase inhibitor 3; STAT3, signal transducer and activator of transcription 3 .

generation] and fine-tuned HIF-1 $\alpha$ levels in HCC cells during hypoxia via a miR-mediated negative-feedback mechanism. In addition, p65 can positively regulate HIF- $1 \alpha$ expression in HCC cells during hypoxia by interacting with signal transducer and activator of transcription 3 (STAT3) (Won et al., 2015).
Pyruvate kinase (PK) is involved in glycolysis and limits the rate of aerobic glycolysis. Human PK genes (L, R, M1, and M2 isoforms) include PKLR and PKM (Mazurek, 2011). It is known that PKM2 is overexpressed in many cancer cell lines, where it promotes aerobic glycolysis. Recently, Dong et al. (2015) demonstrated that PKM2 was overexpressed in HCC 


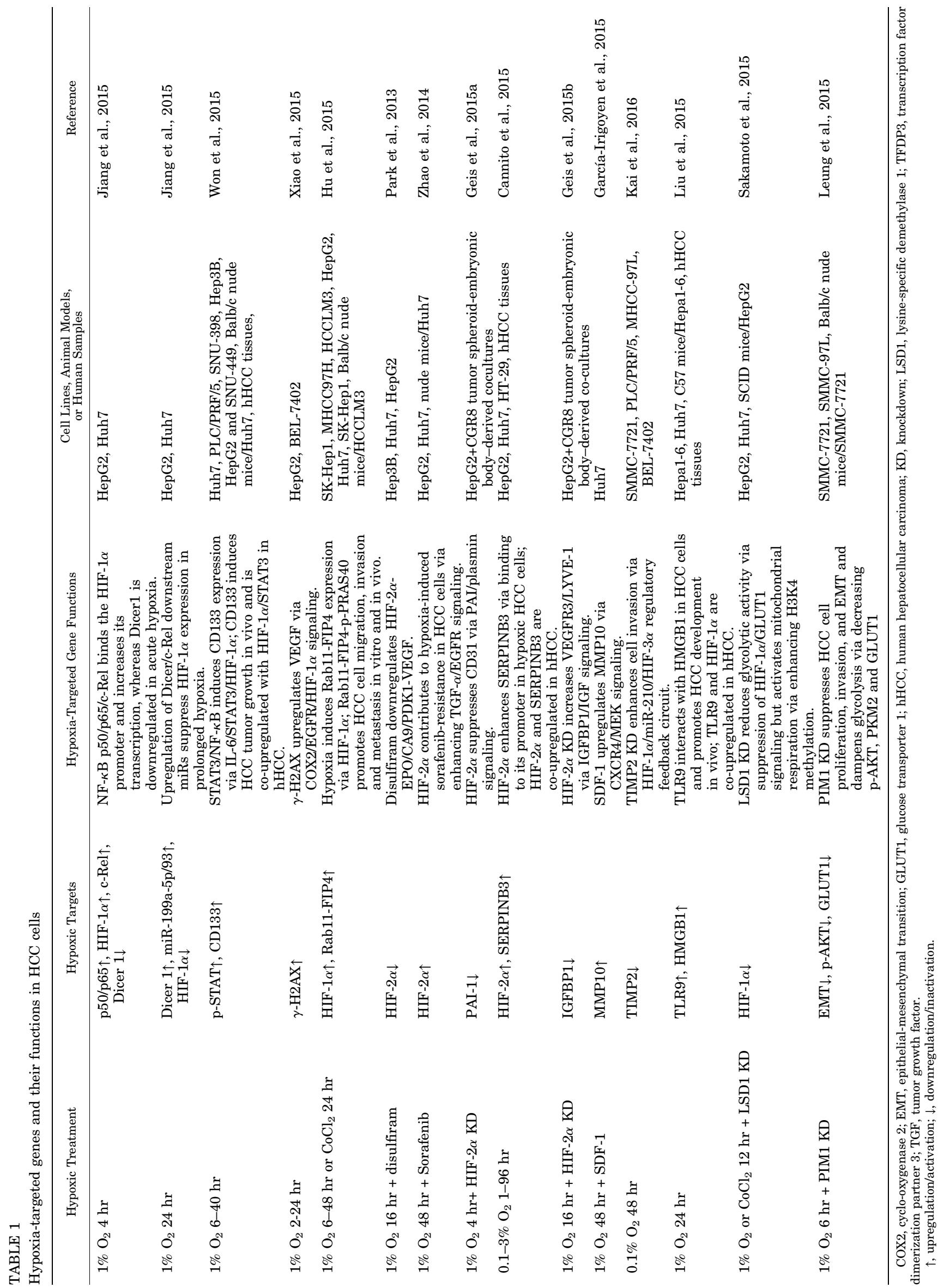


samples. Further, these authors showed that PKM2 can phosphorylate STAT3 at tyrosine 705, which further upregulates HIF- $1 \alpha$ and promotes HCC cell proliferation. This evidence suggests a molecular link between glucose metabolism and hypoxic responses involving PKM2-STAT3-HIF-1 $\alpha$ signaling. Phosphorylated H2A histone family, member $\mathrm{X}$ $(\gamma-\mathrm{H} 2 \mathrm{AX})$ is a common indicator of DNA damage/repair in HCC (Liu et al., 2012a; Matsuda et al., 2013). Xiao et al. (2015) demonstrated that $\gamma$-H2AX was induced in HCC cells upon hypoxia. Further, the increased $\gamma$-H2AX expression correlated to larger HCC tumor sizes, advanced TNM stages, and poor OS in HCC after liver transplantation. Knockingdown $\gamma$-H2AX effectively suppressed mRNA levels for cyclooxygenase 2 and epidermal growth factor receptor (EGFR) in BEL-7402 and HepG2 cells, and partially suppressed HIF-1 $\alpha$ and VEGF expression upon hypoxia. Further, knockdown of EGFR effectively downregulated HIF-1 $\alpha$ and VEGF in hypoxic HCC cells. These results suggest that $\gamma$-H2AX regulates hypoxic responses in HCC cells via an EGFR-HIF-1 $\alpha-\mathrm{VEGF}$ axis. Clinically, higher $\gamma$-H2AX expression combined with HIF- $1 \alpha$ and EGFR provided a more valuable predictor for patients with poor HCC prognosis after liver transplantation.

Rab11-FIP4 (Rab11-family interacting protein) probably functions as a downstream target of HIF- $1 \alpha$ in hypoxic HCC cells. In HCC samples, Rab11-FIP4 expression was positively related to HIF-1 $\alpha$ expression (Hu et al., 2015). Clinical data revealed that HIF-1 $\alpha$ combined with Rab11-FIP4 indicates poor prognosis more reliably. Mechanistically, Rab11-FIP4 promoted PRAS40 (proline-rich Akt substrate) expression, HCC cell mobility/invasion, and remote lung metastasis of HCC cells in mice. Considering that PRAS40 is a key downstream target of the mTOR, HIF- $1 \alpha$ may play a role in regulating autophagy via the Rab11-FIP4/mTOR-PRAS40 pathway in HCC cells in response to hypoxia.

\section{Role of HIF-2 $\alpha$ in HCC}

Compared with HIF- $1 \alpha$, HIF-2 $\alpha$ has been less studied in HCC. A recent meta-analysis of 1066 Chinese HCC patients from seven independent studies showed that higher HIF-2 $\alpha$ correlated well to the increased capsule infiltration/vein invasion/histologic grade but not with HCC prognosis (Yao et al., 2015). HIF- $2 \alpha$ and HIF- $1 \alpha$ were prominently upregulated in $\mathrm{HCC}$ cells upon 16 hours of hypoxic $\left(1 \% \mathrm{O}_{2}\right)$ incubation (Park et al., 2013). Knockdown of HIF-2 $\alpha$ enhanced autophagic activity, attenuated apoptosis (Menrad et al., 2010), and enhanced the effects of doxorubicin (He et al., 2012) or sorafenib (Zhao et al., 2014) on suppressing the development of HCC xenografts in mice. These findings suggest an oncogenic effect of HIF-2 $\alpha$ in HCC cells. However, there are also studies (Sun et al., 2013; Yang et al., 2016) reporting that overexpression of HIF-2 $\alpha$ induced higher levels of apoptosis and inhibited HCC tumor growth in mice, suggesting a tumor suppressor function for HIF-2 $\alpha$ in HCC. Yang et al. (2016) reported that HIF- $2 \alpha$ was decreased in HCC lesions compared with peritumoral tissues $(n=206)$ and that patients with high HIF-2 $\alpha$ had longer OS. A previous study (Menrad et al., 2010) showed that knockdown of HIF- $1 \alpha$ increased HIF- $2 \alpha$ and that knockdown of HIF- $2 \alpha$ increased HIF- $1 \alpha$ in HCC cells, suggesting a balance between HIF- $1 \alpha$ and HIF- $2 \alpha$ in HCC cells. Sun et al. (2013) reported that transcription factor dimerization partner 3 (TFDP3) was a downstream target of HIF-2 $\alpha$ in HCC cells. Knockdown of HIF-2 $\alpha$ downregulated the expression of VEGF/cyclin D1/tumor growth factor $\alpha$ and inhibited EGFR activation (Zhao et al., 2014). Geis et al. (2015a) identified plasminogen activator inhibitor 1 (PAI-1) as an HIF-2 $\alpha$ targeted gene in HepG2 cells by microarray assay. Cannito et al. (2015) demonstrated that HIF- $2 \alpha$ but not HIF- $1 \alpha$ bound to the promoter of SERPINB3 gene (a cysteine-proteases inhibitor) and upregulated SERPINB3 gene expression in hypoxic HCC cells. In addition, insulin-like growth factor binding protein 1 (IGFBP1) was identified as an HIF-2 $\alpha$ targeted gene that links HIF-2 $\alpha$ to insulin-like growth factor (IGF) signaling (Geis et al., 2015b). Taken together, HIF-2 $\alpha$ plays diverse roles in regulating hypoxic responses of $\mathrm{HCC}$ cells, distinct from those of $\mathrm{HIF}-1 \alpha$, in certain cellular contexts.

\section{Role of MMPs in HCC}

MMPs are key molecular players in regulating tumor microenvironment (Kessenbrock et al., 2010). Several members of the MMP family (including MMPs 1, 2, 3, and 9) were upregulated in human HCC tissues (Okazaki and Inagaki, 2012), which contributed to the migration/invasion of HCC in vitro (Chen et al., 2013). Recently, MMP-8 and MMP-10 were also found to be highly associated with human HCC. Qin et al. (2016) reported that MMP-8 was coexpressed with tumor growth factor $\beta 1$ in highly aggressive-HCC patients. García-Irigoyen et al. (2015) reported that MMP10 was upregulated in both human and diethylnitrosamine-induced mouse hepatoma. In the MMP10-knockout mice treated with diethylnitrosamine, not only was the incidence of HCC decreased, but the tumor size, vascularization, and lung metastasis were also reduced. During hypoxia, MMP10 was upregulated in HCC cells via Erk-mediated signaling pathways (García-Irigoyen et al., 2015). In addition, MMP10 could be upregulated by carboxylic acid-truncated hepatitis B virus $\mathrm{X}$ protein $(\mathrm{HBx} \Delta \mathrm{C} 1)$ in $\mathrm{HCC}$ cells (Sze et al., 2013). Mutation of $\mathrm{HBx} \Delta \mathrm{C} 1$ at its binding sites for the MMP10 promoter abolished the effect of $\mathrm{HBx} \Delta \mathrm{C} 1$ on MMP10 induction. Silencing MMP10 in $\mathrm{HBx} \Delta \mathrm{C} 1$-expressing HepG2 cells inhibited cell migration/invasion. Since $\mathrm{HBx} \Delta \mathrm{C} 1$ is expressed in $46 \%$ of $\mathrm{HCC}$ and is highly associated with tumor invasion, $\mathrm{HBx} \Delta \mathrm{C} 1-\mathrm{MMP} 10$ signaling pathway may be crucial for HCC progression.

Tissue inhibitor of metalloproteinases-2 (TIMP2) is consistently downregulated in human HCC lesions; decreased TIMP2 is associated with liver invasion and poorer patient survival (Kai et al., 2016). Mechanistically, TIMP2 suppression is controlled by an HIF- $1 \alpha / \mathrm{miR}-210 / \mathrm{HIF}-3 \alpha$ feedback circuit in hypoxic HCC cells.

\section{Role of HMGB1 in HCC}

HMGB1 is a nuclear damage-associated molecule, induced upon hypoxia, that is associated with HCC invasion and metastasis. Meta-analysis showed a significant correlation between higher HMGB1 and poorer OS and progression-free survival in HCC (Wu et al., 2016). Yan et al. (2012) reported that hypoxia-induced extracellular release of HMGB1 can activate cysteinyl aspartate-specific proteinase-1 (caspase-1) via Toll-like receptor 4 (TLR4). Further, caspase-1 promoted interleukin (IL)-1 $\alpha / 18$ cleavage and release. Overexpression of HMGB1 or administration of recombinant HMGB1 enhanced 
HCC cell invasion, whereas knockdown of HMGB1 inhibited invasion and pulmonary metastasis of HCC in mice. Recently, Chen et al. (2015) reported that HMGB1 promoted HCC progression by upregulating miR-21 and then subsequently suppressing MMP inhibitors [reversion-inducing cysteinerich protein with kazal motifs (RECK) and TIMP3] in an IL6/STAT3-dependent manner. The results of these studies suggest that hypoxia promotes HCC invasion and metastasis by linking HMGB1 to the TLR4-mediated proinflammatory signaling pathway.

HMGB1 can also promote HCC growth by binding to mitochondrial DNA (mtDNA) and activating TLR9-mediated signaling (Liu et al., 2015). These authors showed that, in hypoxic HCC cells, nuclear HMGB1 was translocated to the cytoplasm and bound to free mtDNA, which further activated TLR9. The activation of HMGB1, mtDNA, and TLR9 depended on each other and was crucial for HCC cell proliferation in vitro and for HCC development in vivo.

\section{Role of Beclin 1 in HCC}

Beclin-1, a marker of autophagy, is altered in various cancers, including HCC. Qiu et al. (2014) reported that Beclin-1 expression was strong in 13/22 (59.1\%), moderate in $15 / 53(28.3 \%)$, and weak or negative in $7 / 28(14.6 \%)$ HCC specimens. Compared with nontumor adjacent tissues $(n=$ 57), Beclin-1 in HCC lesions $(n=103)$ was decreased significantly (Qiu et al., 2014). Reduced Beclin-1 correlated with cirrhosis, Edmondson grade, vascular invasion, microvessel density, and other molecular markers [such as PCNA (proliferating cell nuclear antigen), B-cell leukemia/lymphoma (Bcl)-2, and neuroepithelial cell transforming 1 (NET-1)] but was negatively correlated with BCL-2-associated X, apoptosis regulator (Bax) levels. Increased 5-year OS in HCC correlated to higher Beclin-1 levels with lower PCNA/Bcl-2/NET-1 or higher Bax. Thus, Beclin-1 could be regarded as an independent prognostic marker in HCC (Qiu et al., 2014).

Osman et al. (2015) investigated the role of Beclin-1 in HCC $(n=65)$. Beclin-1 was decreased in nearly half of HCC specimens (49.2\%). Increased Beclin-1 was found mainly in cases with viral infection or higher HIF- $1 \alpha(64.6 \%)$. On the basis of HIF- $1 \alpha$ expression, the high or low HIF- $1 \alpha$ group was divided, representing either hypoxia or normoxia cases. In the high HIF-1 $\alpha$ group, increased Beclin-1 correlated well to many tumor parameters (i.e., grade, stage, size, multifoci). However, in the low-HIF- $1 \alpha$ group, an association of Beclin-1 and tumor parameters was not evident. These results support a conclusion that coexpression of Beclin-1 and HIF- $1 \alpha$ is associated with HCC progression.

\section{Alteration of Glucose Metabolism in HCC}

To adapt to an hypoxic microenvironment, most cancer cells undergo a mitochondrial-glycolytic metabolism shift. This is a hallmark of cancers, but the underlying molecular mechanism remains poorly understood. Sakamoto et al. (2015) reported that suppression of lysine-specific demethylase1 (LSD1) downregulated HIF-1 $\alpha$ /glucose transporter 1(GLUT1) and other glycolytic enzymes but upregulated a set of mitochondrial metabolism genes. LSD1 and GLUT1 were consistently co-overexpressed in human HCC tissues. These findings suggest that LSD1 is required for the glycolytic-mitochondrial metabolism shift in HCC cells. Leung et al. (2015) reported that PIM1, a serine/threonine kinase, was overexpressed in $39 \%(n=56)$ of human primary HCC cases. Hypoxia $\left(1 \% \mathrm{O}_{2}\right)$ significantly enhanced PIM1 expression in HCC cell lines. Knockdown of PIM1 reduced glucose uptake and suppressed HCC growth and metastasis in vivo. This evidence suggests that hypoxia-induced PIM1 is important for the mitochondrial-glycolytic metabolism shift in HCC. Jia et al. (2016) reported that miR-592 was downregulated in HCC specimens. These authors showed that overexpression of miR-592 reduced HIF- $1 \alpha$, glycolytic metabolism, and HCC growth. In HCC cases, reduced miR-592 was associated with various malignant parameters or poorer OS.

\section{Role of Neuroglobin in HCC}

Hypoxia plays a fundamental role in carcinogenesis; however, the underlying mechanisms remain far from clear. An important reason might be the lack of an intracellular $\mathrm{O}_{2}$ acceptor. The discovery of two intracellular members of the hemoglobin family, neuroglobin ( $\mathrm{Ngb}$ ) and cytoglobin (Cygb) in mammalian cells provides novel insights for investigating hypoxia-mediated responses in various diseases, including cancers (Qiu and Chen, 2014).

Neuroglobin, a monomeric heme-containing globin $(17 \mathrm{kDa})$, exists predominantly in cells and tissues with a high metabolic rate, such as neurons/brain, endocrine gland cells, and liver (Qiu and Chen, 2014). The oxygen-binding affinity of NGB (1-2 torr) is similar to that of myoglobin and is much higher than that of hemoglobin (12 torr) (Qiu and Chen, 2014). However, the hexa-coordinated nature of the $\mathrm{Fe}^{2+}$ in the heme of NGB makes it difficult for the protein to release $\mathrm{O}_{2}$ under physiologic conditions. It is generally hypothesized that Ngb may serve as an $\mathrm{O}_{2} / \mathrm{ROS}$ sensor/carrier or NO/ROS scavenger (Ascenzi et al., 2014; Burmester and Hankeln, 2014; Qiu and Chen, 2014; Cai et al., 2016; Reuss et al., 2016).

Most previous studies reveal that Ngb can be upregulated in neural cells by acute hypoxia/ischemia, and that Ngb plays a protective role against ischemia (Sun et al., 2001; Chen et al., 2005) or oxidative stress (Ye et al., 2009; Antao et al., 2010). Recently, the expression of Ngb and its homologous Cygb were also investigated in various cancers. Emara et al. $(2010,2014)$ reported that $\mathrm{Ngb} / \mathrm{Cygb}$ was widely expressed in many cancers through microarray analysis. However, Gorr et al. (2011) reported that Ngb, but not Cygb, was elevated in only a few types of tumors. The inconsistency of Ngb expression in cancers was also noted in other studies. For example, Shivapurkar et al. (2008) reported a decrease of Ngb, whereas Oleksiewicz et al. (2011) reported an elevation of Ngb, in lung cancers. This discrepancy might be the result of using different Ngb antibodies, or the differential expression of Ngb depending on specific cellular contexts (e.g., acute or chronic hypoxia, Ngb promoter hypermethylation). We had detected Ngb in liver, breast, lung, bladder, kidney, pancreas, and colon cancers and found a significant decrease of $\mathrm{Ngb}$ in HCC specimens only $(P<0.0001$ versus adjacent nontumor tissues/normal liver tissues) (Zhang et al., 2013). Consistently, the level of Ngb mRNA was also decreased in HCC lesions. The downregulation of Ngb in HCC is in accordance with the alteration of Ngb level in neurons during chronic hypoxia (Hota et al., 2012; Liu et al., 2012b), suggesting that chronic hypoxia might be a major factor controlling Ngb expression in 
HCC cells. In addition, hormones such as $17 \beta$-estradiol may also regulate Ngb expression in HCC (Fiocchetti et al., 2014).

Further studies revealed that Ngb suppressed HCC proliferation in vivo and in vitro (Zhang et al., 2013). These investigators found that Ngb overexpression significantly suppressed HepG2 cell proliferation and colony formation on soft agar and prevented the G0/G1-S transition, whereas knockdown of Ngb showed opposite effects. Moreover, Ngb overexpression suppressed the growth of HCC xenografts and reduced the tumor weight in nude mice. Mechanistically, Ngb bound to proto-oncogene serine/threonine-protein kinase (Raf)-1 and suppressed Raf-MEK-Erk in HCC cells. Interestingly, mutation of the oxygen-binding sites of Ngb (His-64) evidently altered its interaction with Raf-1 and the resulting Erk phosphorylation. Therefore, we propose that Ngb directly links the $\mathrm{O}_{2}$ signal to the Raf-MEK-Erk pathway (Fig. 1). Considering that Raf is an important therapeutic target of sorafenib for HCC, targeting Ngb-Raf interactions might treat $\mathrm{HCC}$ in the future. In addition to Raf-1, Ngb also binds to other proteins such as phosphatase and tensin homolog (PTEN), AKT, G protein, cytochrome C, and 14-3-3 proteins, which are important signaling proteins controlling cell survival, death, and proliferation. It is conceivable that additional binding partners of Ngb will be identified. The binding affinity of Ngb to its client proteins is regulated by its $\mathrm{O}_{2}$-binding site, suggesting that $\mathrm{Ngb}$ directly links $\mathrm{O}_{2}$ signals to intracellular signaling pathways. This function of $\mathrm{Ngb}$ as a signaling protein is distinct from that of HIF as a transcriptional factor, providing a novel point for investigating the effects and mechanisms of hypoxia in cancers.

\section{Targeting Hypoxia for HCC Therapy}

Role of YQ23. YQ23 is a synthetic tetrameric hemoglobin that facilitates oxygen delivery (Ling et al., 2014). YQ23 is taken up into cells by receptor(s)-mediated endocytosis (Man and Yau, 2015). Li et al. (2014) reported that the administration of YQ23 effectively decreased HCC incidence in an orthotopic rat HCC model. In addition, YQ23 reduced metastatic numbers and size in the lung following liver ischemia/ reperfusion or hepatectomy ( $\mathrm{Li}$ et al., 2014). Mechanistically, YQ23 inhibited HIF-1 $\alpha$-dependent angiogenesis in combination with other anticancer drugs (Man and Yau, 2015). In addition, YQ23 suppressed the mobilization of endothelial progenitor cells via the C-X-C motif chemokine 10 (CXCL10)/ CXCR3-tumor necrosis factor $\alpha /$ IL6 signaling pathway (Ling et al., 2014). The data support the possible usefulness of YQ23 for HCC therapy by its promotion of HCC cell oxygenation.

\section{Role of Sorafenib}

Sorafenib, a tyrosine kinase inhibitor, is a multitargeted agent and the only effective first-line drug for advanced HCC patients. However, drug-resistance can develop via hypoxiamediated mechanisms (Llovet et al., 2008). Ma et al. (2014) showed that sorafenib upregulated HIF-2 $\alpha / \mathrm{VEGF} /$ cyclin D1 but downregulated HIF-1 $\alpha$. Knockdown of HIF-2 $\alpha$ enhanced the therapeutic results of sorafenib in HCC xenograft models (Liu et al., 2015). Chen et al. (2014) reported that sorafenib upregulated stromal cell-derived-1 (SDF1 $\alpha$ ) and CXCR4 in hypoxic HCC cells and suppressed SDF1 $\alpha /$ CXCR4 signaling, resuming the anticancer effect of sorafenib despite the presence of hypoxia. Further, suppression of the SDF1 $\alpha$ CXCR4 signaling pathway significantly enhanced the therapeutic effects of sorafenib in orthotopic HCC tumors in mice upon hypoxia (Hato et al., 2014). Clinically, sorafenib in combination with or following transarterial chemoembolization (TACE) resulted in longer OS in HCC patients with portal vein invasion than did sorafenib monotherapy (Ha et al., 2016). Together, these results suggest that hypoxia significantly affects sorafenib therapy. Since hypoxia is heavily involved in sorafenib resistance in HCC therapy, it will be interesting to test whether the combination of sorafenib and YQ23 may have synergistic therapeutic effects for HCC. Theoretically, YQ23 cannot only promote tumor oxygenation but also suppress angiogenesis or inflammation via VEGF- or CXCR-signaling pathways in HCC.

\section{Roles of Other Drugs}

2-Methoxyestradiol (2-ME2) is a promising cancer therapeutic drug that downregulates HIF-1/2. Ma et al. (2014) reported that 2-ME2 effectively downregulated HIF- $1 \alpha / \mathrm{HIF}-2 \alpha$, cyclin D1, VEGF, and lactate dehydrogenase A in HCC cells, which contributed to the resistance of the cells to hypoxia. Further, 2-ME2 in combination with sorafenib yielded better anticancer results in HCC by suppressing tumor angiogenesis. Celastrol is another promising anticancer drug that inhibits the hypoxia-induced HIF-1 pathway in HCC cells (Ma et al., 2014). In this study, celastrol prevented HCC growth by downregulating erythropoietin and VEGF, and suppressing mTOR, p70-S6 kinase 1, eIF4E, and Erk signaling.

\section{Conclusion}

Recent evidence supports the conclusion that hypoxia plays an important role in HCC development and therapy. The decrease of $\mathrm{pO}_{2}$ within $\mathrm{HCC}$ lesions suggests that hypoxia plays a pivotal role during HCC development. Hypoxia marker HIF- $1 \alpha$ is a reliable indicator of poor prognosis for HCC patients. Hypoxia promotes HCC development via complicated mechanisms. Neuroglobin represents a novel type of hypoxia sensor in HCC. Improving HCC oxygenation or suppressing hypoxia-induced signaling are potential therapies for HCC.

\section{Authorship Contribution}

Wrote or contributed to the writing of the manuscript: Xiong, Qiu, $\mathrm{Hu}$, Chen.

\section{References}

Antao ST, Duong TT, Aran R, and Witting PK (2010) Neuroglobin overexpression in cultured human neuronal cells protects against hydrogen peroxide insult via activating phosphoinositide-3 kinase and opening the mitochondrial K(ATP) channel. Antioxid Redox Signal 13:769-781.

Arizumi T, Ueshima K, Chishina H, Kono M, Takita M, Kitai S, Inoue T, Yada N, Hagiwara S, Minami Y, et al. (2014) Decreased blood flow after sorafenib administration is an imaging biomarker to predict overall survival in patients with advanced hepatocellular carcinoma. Dig Dis 32:733-739.

Ascenzi P, Gustincich S, and Marino M (2014) Mammalian nerve globins in search of functions. IUBMB Life 66:268-276.

Bayraktutan ÜKantarci A, Ogul H, Kizrak Y, Ozyigit O, Yuceler Z, Genc B, and Ozogul B (2014) Evaluation of hepatocellular carcinoma with computed tomography perfusion imaging. Turk J Med Sci 44:193-196.

Burmester T and Hankeln T (2014) Function and evolution of vertebrate globins. Acta Physiol (Oxf) 211:501-514.

Cai B, Li W, Mao X, Winters A, Ryou MG, Liu R, Greenberg DA, Wang N, Jin K, and Yang SH (2016) Neuroglobin Overexpression Inhibits AMPK Signaling and Promotes Cell Anabolism. Mol Neurobiol 53:1254-1265. 
Cannito S, Turato C, Paternostro C, Biasiolo A, Colombatto S, Cambieri I, Quarta S, Novo E, Morello E, Villano G, et al. (2015) Hypoxia up-regulates SERPINB3 through HIF-2alpha in human liver cancer cells. Oncotarget 6:2206-2221.

Cao S, Yang S, Wu C, Wang Y, Jiang J, and Lu Z (2014) Protein expression of hypoxia-inducible factor-1 alpha and hepatocellular carcinoma: a systematic review with meta-analysis. Clin Res Hepatol Gastroenterol 38:598-603.

Chen BB, Hsu CY, Yu CW, Liang PC, Hsu C, Hsu CH, Cheng AL, and Shih TT (2016) Dynamic contrast-enhanced MR imaging of advanced hepatocellular carcinoma: comparison with the liver parenchyma and correlation with the survival of patients receiving systemic therapy. Radiology 281:454-464.

Chen JS, Huang XH, Wang Q, Huang JQ, Zhang LJ, Chen XL, Lei J, and Cheng ZX (2013) Sonic hedgehog signaling pathway induces cell migration and invasion through focal adhesion kinase/AKT signaling-mediated activation of matrix metalloproteinase (MMP)-2 and MMP-9 in liver cancer. Carcinogenesis 34:10-19.

Chen M, Liu Y, Varley P, Chang Y, He XX, Huang H, Tang D, Lotze MT, Lin J, and Tsung A (2015) High-Mobility Group Box 1 Promotes Hepatocellular Carcinoma Progression through miR-21-Mediated Matrix Metalloproteinase Activity. Cancer Res 75:1645-1656.

Chen XQ, Qin LY, Zhang CG, Yang LT, Gao Z, Liu S, Lau LT, Fung YW, Greenberg DA, and Yu AC (2005) Presence of neuroglobin in cultured astrocytes. Glia 50: 182-186.

Chen Y, Huang Y, Reiberger T, Duyverman AM, Huang P, Samuel R, Hiddingh L, Roberge S, Koppel C, Lauwers GY, et al. (2014) Differential effects of sorafenib on liver versus tumor fibrosis mediated by stromal-derived factor 1 alpha/C-X-C receptor type 4 axis and myeloid differentiation antigen-positive myeloid cell infiltration in mice. Hepatology 59:1435-1447.

Chou CT, Chen RC, Lin WC, Ko CJ, Chen CB, and Chen YL (2014) Prediction of microvascular invasion of hepatocellular carcinoma: preoperative CT and histopathologic correlation. AJR Am J Roentgenol 203:W253-W259.

Dong T, Yan Y, Chai H, Chen S, Xiong X, Sun D, Yu Y, Deng L, and Cheng F (2015) Pyruvate kinase M2 affects liver cancer cell behavior through up-regulation of HIF-1alpha and Bcl-xL in culture. Biomed Pharmacother 69:277-284

Emara M, Turner AR, and Allalunis-Turner J (2010) Hypoxic regulation of cytoglobin and neuroglobin expression in human normal and tumor tissues. Cancer Cell Int 10:33.

Emara M, Turner AR, and Allalunis-Turner J (2014) Adult, embryonic and fetal hemoglobin are expressed in human glioblastoma cells. Int $J$ Oncol 44:514-520.

Fiocchetti M, Nuzzo MT, Totta P, Acconcia F, Ascenzi P, and Marino M (2014) Neuroglobin, a pro-survival player in estrogen receptor alpha-positive cancer cells Cell Death Dis 5:e1449.

Fukuda K, Taniguchi H, Koh T, Kunishima S, and Yamagishi H (2004) Relationships between oxygen and glucose metabolism in human liver tumours: positron emission tomography using (15)O and (18)F-deoxyglucose. Nucl Med Commun 25 $577-583$

Gaballah HH, Zakaria SS, and Ismail SA (2014) Activity and expression pattern of NF-kappaB /P65 in peripheral blood from hepatocellular carcinoma patients - link to hypoxia inducible factor -1alpha. Asian Pac J Cancer Prev 15:6911-6917.

García-Irigoyen O, Latasa MU, Carotti S, Uriarte I, Elizalde M, Urtasun R, Vespasiani-Gentilucci U, Morini S, Benito P, Ladero JM, et al. (2015) Matrix metalloproteinase 10 contributes to hepatocarcinogenesis in a novel crosstalk with the stromal derived factor 1/C-X-C chemokine receptor 4 axis. Hepatology 62: $166-178$

Geis T, Doring C, Popp R, Grossmann N, Fleming I, Hansmann ML, Dehne N, and Brune B (2015a) HIF-2alpha-dependent PAI-1 induction contributes to angiogenesis in hepatocellular carcinoma. Exp Cell Res 331:46-57.

Geis T, Popp R, Hu J, Fleming I, Henke N, Dehne N, and Brune B (2015b) HIF-2alpha attenuates lymphangiogenesis by up-regulating IGFBP1 in hepatocellular carcinoma. Biol Cell 107:175-188.

Gorr TA, Wichmann D, Pilarsky C, Theurillat JP, Fabrizius A, Laufs T, Bauer T, Koslowski M, Horn S, Burmester T, et al. (2011) Old proteins - new locations: myoglobin, haemoglobin, neuroglobin and cytoglobin in solid tumours and cancer cells. Acta Physiol (Oxf) 202:563-581.

Guo M and Yu Y (2014) Application of 128 slice 4D CT whole liver perfusion imaging in hepatic tumor. Cell Biochem Biophys 70:173-178.

Ha Y, Lee D, Shim JH, Lim YS, Lee HC, Chung YH, Lee YS, Park SR, Ryu MH, Ryoo BY, et al. (2016) Role of transarterial chemoembolization in relation with sorafenib for patients with advanced hepatocellular carcinoma. Oncotarget 7:74303-74313.

Hao XJ, Li JP, Jiang HJ, Li DQ, Ling ZS, Xue LM, and Feng GL (2013) CT assessment of liver hemodynamics in patients with hepatocellular carcinoma after argonhelium cryoablation. Hepatobiliary Pancreat Dis Int 12:617-621.

Hato T, Goyal L, Greten TF, Duda DG, and Zhu AX (2014) Immune checkpoint blockade in hepatocellular carcinoma: current progress and future directions. Hepatology 60:1776-1782.

Hayano K, Lee SH, Yoshida H, Zhu AX, and Sahani DV (2014) Fractal analysis of CT perfusion images for evaluation of antiangiogenic treatment and survival in hepatocellular carcinoma. Acad Radiol 21:654-660.

He C, Sun XP, Qiao H, Jiang X, Wang D, Jin X, Dong X, Wang J, Jiang H, and Sun X (2012) Downregulating hypoxia-inducible factor-2alpha improves the efficacy of doxorubicin in the treatment of hepatocellular carcinoma. Cancer Sci 103:528-534.

Höckel M and Vaupel P (2001) Tumor hypoxia: definitions and current clinical, biologic, and molecular aspects. $J$ Natl Cancer Inst 93:266-276.

Hota KB, Hota SK, Srivastava RB, and Singh SB (2012) Neuroglobin regulates hypoxic response of neuronal cells through Hif-1alpha- and Nrf2-mediated mechanism. J Cereb Blood Flow Metab 32:1046-1060.

Hu F, Deng X, Yang X, Jin H, Gu D, Lv X, Wang C, Zhang Y, Huo X, Shen Q, et al. (2015) Hypoxia upregulates Rab11-family interacting protein 4 through HIF-1alpha to promote the metastasis of hepatocellular carcinoma. Oncogene $\mathbf{3 4}$ $6007-6017$.

Huang WJ, Jeng YM, Lai HS, Fong IU, Sheu FY, Lai PL, and Yuan RH (2015) Expression of hypoxic marker carbonic anhydrase IX predicts poor prognosis in resectable hepatocellular carcinoma. PLoS One 10:e119181.
Ippolito D, Fior D, Bonaffini PA, Capraro C, Leni D, Corso R, and Sironi S (2014) Quantitative evaluation of CT-perfusion map as indicator of tumor response to transarterial chemoembolization and radiofrequency ablation in HCC patients. Eur J Radiol 83:1665-1671.

James PE, Madhani M, Roebuck W, Jackson SK, and Swartz HM (2002) Endotoxininduced liver hypoxia: defective oxygen delivery versus oxygen consumption. Nitric Oxide 6:18-28.

Jia YY, Zhao JY, Li BL, Gao K, Song Y, Liu MY, Yang XJ, Xue Y, Wen AD, and Shi L (2016) miR-592/WSB1/HIF-1alpha axis inhibits glycolytic metabolism to decrease hepatocellular carcinoma growth. Oncotarget 7:35257-35269.

Jiang Y, Zhu Y, Wang X, Gong J, Hu C, Guo B, Zhu B, and Li Y (2015) Temporal regulation of HIF-1 and NF-kappaB in hypoxic hepatocarcinoma cells. Oncotarget 6:9409-9419.

Jungermann K and Kietzmann T (2000) Oxygen: modulator of metabolic zonation and disease of the liver. Hepatology 31:255-260.

Kai AK, Chan LK, Lo RC, Lee JM, Wong CC, Wong JC, and Ng IO (2016) Downregulation of TIMP2 by HIF-1alpha/miR-210/HIF-3alpha regulatory feedback circuit enhances cancer metastasis in hepatocellular carcinoma. Hepatology 64 473-487.

Kessenbrock K, Plaks V, and Werb Z (2010) Matrix metalloproteinases: regulators of the tumor microenvironment. Cell 141:52-67.

Lai JP, Conley A, Knudsen BS, and Guindi M (2015) Hypoxia after transarterial chemoembolization may trigger a progenitor cell phenotype in hepatocellular carcinoma. Histopathology 67:442-450.

Leung CO, Wong CC, Fan DN, Kai AK, Tung EK, Xu IM, Ng IO, and Lo RC (2015) PIM1 regulates glycolysis and promotes tumor progression in hepatocellular carcinoma. Oncotarget 6:10880-10892.

Li CX, Wong BL, Ling CC, Ma YY, Shao Y, Geng W, Qi X, Lau SH, Kwok SY, Wei N, et al. (2014) A novel oxygen carrier "YQ23" suppresses the liver tumor metastasis by decreasing circulating endothelial progenitor cells and regulatory $\mathrm{T}$ cells. $B M C$ Cancer 14:293.

Li W, Wang R, Xie H, Zhang J, and Jia Z (2015) Changes of pathological and physiological indicators affecting drug metabolism in rats after acute exposure to high altitude. Exp Ther Med 9:98-104

Ling CC, Ng KT, Shao Y, Geng W, Xiao JW, Liu H, Li CX, Liu XB, Ma YY, Yeung WH, et al. (2014) Post-transplant endothelial progenitor cell mobilization via CXCL10/CXCR3 signaling promotes liver tumor growth. J Hepatol 60:103-109.

Liu F, Dong X, Lv H, Xiu P, Li T, Wang F, Xu Z, and Li J (2015) Targeting hypoxia-inducible factor-2alpha enhances sorafenib antitumor activity via betacatenin/C-Myc-dependent pathways in hepatocellular carcinoma. Oncol Lett 10: $778-784$.

Liu J, Hou M, Yuan T, Yi G, Zhang S, Shao X, Chen J, Jia X, and He Z (2012a) Enhanced cytotoxic effect of low doses of metformin combined with ionizing radiation on hepatoma cells via ATP deprivation and inhibition of DNA repair. Oncol Rep 28:1406-1412

Liu N, Yu Z, Xiang S, Zhao S, Tjarnlund-Wolf A, Xing C, Zhang J, and Wang X (2012b) Transcriptional regulation mechanisms of hypoxia-induced neuroglobin gene expression. Biochem J 443:153-164.

Llovet JM, Ricci S, Mazzaferro V, Hilgard P, Gane E, Blanc JF, de Oliveira AC, Santoro A, Raoul JL, Forner A, et al. (2008) Sorafenib in advanced hepatocellular carcinoma. N Engl J Med 359:378-390.

Ma J, Han LZ, Liang H, Mi C, Shi H, Lee JJ, and Jin X (2014) Celastrol inhibits the HIF-1alpha pathway by inhibition of mTOR/p70S6K/eIF4E and ERK1/2 phosphorylation in human hepatoma cells. Oncol Rep 32:235-242.

Man C and Yau T (2015) P6.02* Therapeutic strategies of the investigational new drug, YQ23, for overcoming chemoresistance in hypoxic solid tumours. Ann Oncol 26 (Suppl 2):ii28, doi:10.1093/annonc/mdv095.2.

Matsuda Y, Wakai T, Kubota M, Osawa M, Takamura M, Yamagiwa S, Aoyagi Y, Sanpei A, and Fujimaki S (2013) DNA damage sensor gamma -H2AX is increased in preneoplastic lesions of hepatocellular carcinoma. Sci World J 2013:597095.

Mazurek S (2011) Pyruvate kinase type M2: a key regulator of the metabolic budget system in tumor cells. Int J Biochem Cell Biol 43:969-980.

McGuire S (2016) World Cancer Report 2014. Geneva, Switzerland: World Health Organization, International Agency for Research on Cancer, WHO Press, 2015 Adv Nutr 7:418-419.

Menrad H, Werno C, Schmid T, Copanaki E, Deller T, Dehne N, and Brune B (2010) Roles of hypoxia-inducible factor-1alpha (HIF-1alpha) versus HIF-2alpha in the survival of hepatocellular tumor spheroids. Hepatology 51:2183-2192.

Møller S, Hillingso J, Christensen E, and Henriksen JH (1998) Arterial hypoxaemia in cirrhosis: fact or fiction? Gut 42:868-874

Nishida N and Kudo M (2013) Oxidative stress and epigenetic instability in human hepatocarcinogenesis. Dig Dis 31:447-453.

Okazaki I and Inagaki Y (2012) Novel strategies for hepatocellular carcinoma based on MMPs science. Anticancer Agents Med Chem 12:753-763.

Oleksiewicz U, Daskoulidou N, Liloglou T, Tasopoulou K, Bryan J, Gosney JR, Field JK, and Xinarianos G (2011) Neuroglobin and myoglobin in non-small cell lung cancer: expression, regulation and prognosis. Lung Cancer 74:411-418.

Osman NA, Abd ED, and Kamal IM (2015) Defective Beclin-1 and elevated hypoxiainducible factor (HIF)-1alpha expression are closely linked to tumorigenesis, differentiation, and progression of hepatocellular carcinoma. Tumour Biol 36: 4293-4299.

Park HJ, Kim MS, Cho K, Yun JH, Choi YJ, and Cho CH (2013) Disulfiram deregulates HIF-alpha subunits and blunts tumor adaptation to hypoxia in hepatoma cells. Acta Pharmacol Sin 34:1208-1216.

Qin G, Luo M, Chen J, Dang Y, Chen G, Li L, Zeng J, Lu Y, and Yang J (2016) Reciprocal activation between MMP-8 and TGF-beta1 stimulates EMT and malignant progression of hepatocellular carcinoma. Cancer Lett 374:85-95.

Qiu DM, Wang GL, Chen L, Xu YY, He S, Cao XL, Qin J, Zhou JM, and Zhang YX and E Q (2014) The expression of beclin-1, an autophagic gene, in hepatocellular carcinoma associated with clinical pathological and prognostic significance. $B M C$ Cancer 14:327. 
Qiu XY and Chen XQ (2014) Neuroglobin - recent developments. Biomol Concepts 5: 195-208.

Reuss S, Banica O, Elgurt M, Mitz S, Disque-Kaiser U, Riemann R, Hill M, Jaquish DV, Koehrn FJ, Burmester T, et al. (2016) Neuroglobin Expression in the Mammalian Auditory System. Mol Neurobiol 53:1461-1477.

Reynolds TY, Rockwell S, and Glazer PM (1996) Genetic instability induced by the tumor microenvironment. Cancer Res 56:5754-5757.

Riedl CC, Brader P, Zanzonico PB, Chun YS, Woo Y, Singh P, Carlin S, Wen B, Ling $\mathrm{CC}$, Hricak H, et al. (2008) Imaging hypoxia in orthotopic rat liver tumors with iodine 124-labeled iodoazomycin galactopyranoside PET. Radiology 248:561-570.

Sakamoto A, Hino S, Nagaoka K, Anan K, Takase R, Matsumori H, Ojima H, Kanai Y, Arita K, and Nakao M (2015) Lysine Demethylase LSD1 Coordinates Glycolytic and Mitochondrial Metabolism in Hepatocellular Carcinoma Cells. Cancer Res $\mathbf{7 5}$ 1445-1456.

Severi T, van Malenstein H, Verslype C, and van Pelt JF (2010) Tumor initiation and progression in hepatocellular carcinoma: risk factors, classification, and therapeutic targets. Acta Pharmacol Sin 31:1409-1420.

Shivapurkar N, Stastny V, Okumura N, Girard L, Xie Y, Prinsen C, Thunnissen FB, Wistuba II, Czerniak B, Frenkel E, et al. (2008) Cytoglobin, the newest member of the globin family, functions as a tumor suppressor gene. Cancer Res 68:7448-7456

Siegel R, Ma J, Zou Z, and Jemal A (2014) Cancer statistics, 2014. CA Cancer J Clin 64:9-29.

Srivastava S, Thakkar B, Yeoh KG, Ho KY, Teh M, Soong R, and Salto-Tellez M (2015) Expression of proteins associated with hypoxia and Wnt pathway activation is of prognostic significance in hepatocellular carcinoma. Virchows Arch 466: 541-548.

Sun HX, Xu Y, Yang XR, Wang WM, Bai H, Shi RY, Nayar SK, Devbhandari RP, He YZ, Zhu QF, et al. (2013) Hypoxia inducible factor 2 alpha inhibits hepatocellular carcinoma growth through the transcription factor dimerization partner 3/ E2F transcription factor 1-dependent apoptotic pathway. Hepatology 57:1088-1097.

Sun Y, Jin K, Mao XO, Zhu Y, and Greenberg DA (2001) Neuroglobin is up-regulated by and protects neurons from hypoxic-ischemic injury. Proc Natl Acad Sci USA 98 : 15306-15311.

Sze KM, Chu GK, Lee JM, and Ng IO (2013) C-terminal truncated hepatitis B virus x protein is associated with metastasis and enhances invasiveness by C-Jun/matrix metalloproteinase protein 10 activation in hepatocellular carcinoma. Hepatology 57:131-139.

Taouli B, Johnson RS, Hajdu CH, Oei MT, Merad M, Yee H, and Rusinek H (2013) Hepatocellular carcinoma: perfusion quantification with dynamic contrastenhanced MRI. AJR Am J Roentgenol 201:795-800.

Vaupel P, Hockel M, and Mayer A (2007) Detection and characterization of tumor hypoxia using pO2 histography. Antioxid Redox Signal 9:1221-1236.

Wang B, Ding YM, Fan P, Wang B, Xu JH, and Wang WX (2014) Expression and significance of MMP2 and HIF-1alpha in hepatocellular carcinoma. Oncol Lett 8 $539-546$
Won C, Kim BH, Yi EH, Choi KJ, Kim EK, Jeong JM, Lee JH, Jang JJ, Yoon JH, Jeong WI, et al. (2015) Signal transducer and activator of transcription 3-mediated CD133 up-regulation contributes to promotion of hepatocellular carcinoma. Hepatology 62:1160-1173.

Wu T, Zhang W, Yang G, Li H, Chen Q, Song R, and Zhao L (2016) HMGB1 overexpression as a prognostic factor for survival in cancer: a meta-analysis and systematic review. Oncotarget 7:50417-50427.

Xiao H, Tong R, Cheng S, Lv Z, Ding C, Du C, Xie H, Zhou L, Wu J, and Zheng S (2014) BAG3 and HIF-1 alpha coexpression detected by immunohistochemistry correlated with prognosis in hepatocellular carcinoma after liver transplantation. BioMed Res Int 2014:516518.

Xiao H, Tong R, Ding C, Lv Z, Du C, Peng C, Cheng S, Xie H, Zhou L, Wu J, et al. (2015) gamma-H2AX promotes hepatocellular carcinoma angiogenesis via EGFR/HIF-1alpha/VEGF pathways under hypoxic condition. Oncotarget 6: 2180-2192.

Xu W, Kwon JH, Moon YH, Kim YB, Yu YS, Lee N, Choi KY, Kim YS, Park YK, Kim BW, et al. (2014) Influence of preoperative transcatheter arterial chemoembolization on gene expression in the HIF-1alpha pathway in patients with hepatocellular carcinoma. J Cancer Res Clin Oncol 140:1507-1515.

Yan W, Chang Y, Liang X, Cardinal JS, Huang H, Thorne SH, Monga SP, Geller DA Lotze MT, and Tsung A (2012) High-mobility group box 1 activates caspase-1 and promotes hepatocellular carcinoma invasiveness and metastases. Hepatology 55: 1863-1875.

Yang SL, Liu LP, Niu L, Sun YF, Yang XR, Fan J, Ren JW, Chen GG, and Lai PB (2016) Downregulation and pro-apoptotic effect of hypoxia-inducible factor 2 alpha in hepatocellular carcinoma. Oncotarget 7:34571-34581.

Yao Q, Lv Y, Pan T, Liu Y, Ma J, and Xu G (2015) Prognostic significance and clinicopathological features of hypoxic inducible factor-2alpha expression in hepatocellular carcinoma. Saudi Med J 36:170-175.

Ye SQ, Zhou XY, Lai XJ, Zheng L, and Chen XQ (2009) Silencing neuroglobin enhances neuronal vulnerability to oxidative injury by down-regulating 14-3 3gamma. Acta Pharmacol Sin 30:913-918.

Zhang J, Lan SJ, Liu QR, Liu JM, and Chen XQ (2013) Neuroglobin, a novel intracellular hexa-coordinated globin, functions as a tumor suppressor in hepatocellular carcinoma via Raf/MAPK/Erk. Mol Pharmacol 83:1109-1119.

Zhang Z, Zhang F, Lu Y, and Zheng S (2015) Update on implications and mechanisms of angiogenesis in liver fibrosis. Hepatol Res 45:162-178.

Zhao D, Zhai B, He C, Tan G, Jiang X, Pan S, Dong X, Wei Z, Ma L, Qiao H, et al. (2014) Upregulation of HIF-2alpha induced by sorafenib contributes to the resistance by activating the TGF-alpha/EGFR pathway in hepatocellular carcinoma cells. Cell Signal 26:1030-1039.

Address correspondence to: Dr. Xiao Qian Chen, Hangkong Road 13, Wuhan 430030, China. E-mail: chenxq@mails.tjmu.edu.cn 